\title{
Inhibition of arginine ADP-ribosyltransferase 1 reduces the expression of poly(ADP-ribose) polymerase-1 in colon carcinoma
}

\author{
YI TANG, YA-LAN WANG, LIAN YANG, JIAN-XIA XU, WEI XIONG, MING XIAO and MING LI \\ Department of Pathology, Molecular Medicine and Cancer Research Center, \\ Chongqing Medical University, Chongqing 400016, P.R. China
}

Received February 7, 2013; Accepted April 8, 2013

DOI: $10.3892 / \mathrm{ijmm} .2013 .1370$

\begin{abstract}
Poly(ADP-ribose) polymerase-1 (PARP-1) which mediates poly-ADP-ribosylation, has been extensively investigated in carcinoma compared to arginine ADP-ribosyltransferase 1 (ART1), which mediates mono-ADP-ribosylation. Previous studies have demonstrated that these enzymes promote proliferation and tumor development in colon carcinoma. However, whether there is any association between PARP-1 and ATR1 in colon carcinoma, remains unelucidated. In this study, using immunohistochemical analysis, we detected a higher expression of PARP-1 and ART1 in 63 samples from patients with colon carcinoma compared to 10 samples of normal colonic mucosa; our results revealed a positive correlation between the expression of PARP-1 and ART1 in the 63 human colon carcinoma tissue samples. To determine the correlation between PARP-1 and ART1, inhibitors of PARP-1 and ART1 and lentivirus vector-mediated ART1 short-hairpin RNA (shRNA) were used to culture CT26 murine colon adenocarcinoma cells separately. Using double-label immunofluorescence assay, we detected the expression of PARP-1 in the CT26 cells, which was decreased following treatment with 5-aminoisoquinolinone (5-AIQ, a PARP-1 inhibitor) or meta-iodobenzylguanidine (MIBG, an ART1 inhibitor). However, the expression of ART1 only decreased when the CT26 cells were treated with MIBG. Furthermore, our results demonstrated that silencing ART1 inhibited PARP-1 expression by decreasing the expression of nuclear factor- $\kappa \mathrm{B}(\mathrm{NF}-\kappa \mathrm{B})$, inhibiting ras homolog $\mathrm{A}$ (RhoA). Hence, our data demonstrate the positive correlation between ART1 and PARP-1; the inhibition of ART1 activity downregulates PARP-1 expression by decreasing the activity of NF- $\kappa \mathrm{B}$ in CT26 colon carcinoma cells.
\end{abstract}

Correspondence to: Professor Ya-Lan Wang, Department of Pathology, Molecular Medicine and Cancer Research Center, Chongqing Medical University, No. 1 Medical School Road, Yu Zhong, Chongqing 400016, P.R. China

E-mail: wangyalan074@126.com

Key words: colon carcinoma, arginine ADP-ribosyltransferase, poly(ADP-ribose) polymerase, nuclear factor- $\kappa \mathrm{B}$

\section{Introduction}

ADP-ribosylation plays a role in a number of physiological and pathophysiological processes, including signal transduction, transcription, DNA repair, cell cycle regulation and mitosis, as well as necrosis and apoptosis (1). The classification of ADP-ribosylation includes poly-ADP-ribosylation and mono-ADP-ribosylation. According to the acceptor sites of the different types of mono-ADP ribosylated amino acids, mono-ADP ribosylation has been subdivided into diphthamide-, arginine-, asparagine- and cysteine-specific ADP-ribosylation. In mono-ADP-ribosylation, amino acid ADP-ribosyltransferases (ARTs) are significant catalyzing enzymes which can transfer the ADP-ribose moiety of nicotinamide adenine dinucleotide (NAD) to certain protein amino acids $(2,3)$. ARTs included ART1-7; however, only ART1, -3, -4, and -5 have been verified in the human gemone. In the family of ARTs, ART1 and ART5 are arginine-specific transferases. ART5 has only been detected in humans; however, ART1 has been detected in humans and mice. The function of ART5 has not yet been fully elucidated, but ART1 has been reported to be involved in the inhibitory activity of substrates, including defensin and integrin, and to mediate ADP-ribosylation in humans; therefore, it may participate in the innate immune response $(4,5)$.

Poly(ADP-ribose) polymerase-1 (PARP-1) is an abundant nuclear protein. It belongs to a family of 18 enzymes that cleave $\mathrm{NAD}^{+}$to nicotinamide and ADP-ribose, forming negatively charged long branched (ADP-ribose) polymers on glutamic acid residues of the PARP-1 protein itself and of other acceptor proteins (6). In comparison with ART1, PARP-1 has been investigated in a number of studies on carcinoma. Previously, we demonstrated that the high expression of PARP-1 in human colon carcinoma (7) and the inhibition of PARP-1 by 5-aminoisoquinolinone (5-AIQ) decreases the matrix adhesion, invasion and migration ability of colon carcinoma cells, and suppresses colon carcinoma liver metastasis in mice (8-10).

Previous studies have demonstrated that the ADPribosylation of cell surface proteins mediated by arginine-specific mono-ADP-ribosyltransferase suppresses the proliferation and cytotoxic effector functions of cytotoxic T lymphocytes in vitro $(11,12)$, and prevents proliferation (13) and induces apoptosis in primary T cells (14). As demonstrated in a previous study, in human gastric adenocarcinoma, Helicobacter pylori (H.pylori) 
has enzymatic mono-ADP-ribosyltransferase activity, which enables it to modify membranous proteins and $H$. pylori itself. The authors hypothesized that mono-ADP-ribosyltransferase may contribute to human gastric carcinoma progression; however, this requires fruther clarification (15). In a previous study, we found that the expression of ART1, which positively correlates with the expression of integrin $\alpha \mathrm{V} \beta 3$ and vascular endothelial growth factor (VEGF), was increased in colorectal carcinoma. It has been suggested that ART1 has the ability to promote the formation of blood capillaries in colorectal carcinoma (16).

Previous studies have reported that inhibitors of PARP-1 and arginine-dependent mono-ADP-ribosylation, prevent phenotypic modulation and the proliferation of smooth muscle cells (SMCs) (17), concluding that poly- and mono-ADP-ribosylation are both essential for the SMC response to mitogenic stimulation. High levels of mono- and poly-ADP-ribosylation activity may be necessary for proliferation and differentiation (18-20). Certain studies have revealed opposite effects, according to different cell types, growth conditions, inhibitors and inhibitor concentrations $(21,22)$. Hence, the correlation between PARP-1 and ART1 in colon carcinoma has not yet been elucidated.

$\mathrm{NF}-\kappa \mathrm{B}$, a transcription factor, has been found to mediate various processes, including inflammation, immunity, apoptosis, proliferation, angiogenesis and metastasis $(23,24)$. Of note, certain studies have indicated that $\mathrm{NF}-\kappa \mathrm{B}$ may be regulated by PARP (25) and that NF- $\kappa \mathrm{B}$ may affect the expression of PARP through a feedback mechanism (26).

In this study, meta-iodobenzylguanidine (MIBG), a potent inhibitor of arginine-specific mono-ADP-ribosyltransferases (27), and 5-aminoisoquinolinone (5-AIQ), a water-soluble inhibitor of PARP (28), were used to inhibit the activity of ART1 and PARP, respectively, and the correlation between PARP-1 and ART1 was investigated in colon carcinoma.

\section{Materials and methods}

Tissues and cells. A total of 63 samples of colorectal carcinoma were provided by the Pathology Department of Chongqing Medical University, Chongqing, China. According to the WHO classification stystem for colorectal carcinoma, 2 pathologists diagnosed the tissue samples into grade I (well differentiated), grade II (moderately differentiated), grade III (poorly differentiated). The CT26 cell line, a murine colon adenocarcinoma cell line, was a gift from Professor Yuquan Wei, Sichuan University, Chengdu, China.

Immunochemistry assay. Sliced tissues (4- $\mu$ m-thick) which were fixed by formalin and embedded in paraffin, were successively immersed in different levels of ethanol for hydration. Cell membranes were permeablized with $0.3 \%$ Triton $\mathrm{X}-100$ for $20 \mathrm{~min}$ at $37^{\circ} \mathrm{C}$. After blocking peroxidase activity with $3 \%$ $\mathrm{H}_{2} \mathrm{O}_{2} /$ methanol for $10 \mathrm{~min}$, the specimens were soaked in citrate buffer (ZSGB-Bio, Beijing, China) and heated to $95-98^{\circ} \mathrm{C}$ in a microwave oven (Gree, Zhuhai, China). After cooling to room temperature, the specimens were washed 3 times with phosphate-buffered saline (PBS, ZSGB-Bio) and blocked with confining liquid (5\% bovine serum) (SABC kit; Wuhan Boster Biological Technology, Ltd., Wuhan, China) for $30 \mathrm{~min}$ at $37^{\circ} \mathrm{C}$. All the samples were incubated at $4^{\circ} \mathrm{C}$ overnight with rabbit polyclonal antibody to PARP-1 (Santa Cruz Biotechnology, Santa Cruz, CA, USA) or goat polyclonal antibody to ART1 (Santa Cruz Biotechnology) at dilutions of 1:100. According to the specifications of the SABC kit, the samples were incubated with anti-rabbit antibody and avidin-biotin peroxidase or the anti-goat antibody and avidin-biotin peroxidase, followed by the addition of SABC for $30 \mathrm{~min}$ at $37^{\circ} \mathrm{C}$. After being dyed with diaminobenzidine (DAB) (ZSGB-Bio), the samples were counterstained with hematoxylin in quick succession. For the negative control group, PBS was used instead of the primary antibody.

The staining results were assessed according to the method described in the study by Fromowitz et al $(29,30)$ by 2 independent pathologists as follows: i) positive degree score: 0 , no staining; 1, light yellow; 2, brown; 3, dark brown. ii) Random observations from 5 magnifications $(10 \times 20)$ per field counting 100 tumor cells, the average percentage of positive cells in each field of vision as the percentage of positive cells in the slice. Positive range score: $0,0-5 \% ; 1,6-25 \% ; 2,26-50 \% ; 3,51-75 \%$; $4,>75 \%$. The scores were judged by positive range score plus positive extent score: $<2$, negative $(-) ; 2-3$, slight positive $(+)$; $4-5$, moderately positive $(++) ; 6-7$, strongly positive $(+++)$.

Double-label immunofluorescence assay. The CT26 cells were divided into 3 groups: untreated group, the group treated with $100 \mu \mathrm{M}$ MIBG (Sigma, Ronkonkoma, NY, USA) and the group treated with $100 \mu \mathrm{M}$ 5-AIQ (a gift from Professor M.D. Threadgill, Bath University, Bath, UK). After being treated with $0.5 \%$ Triton for $15 \mathrm{~min}$ and $1 \%$ BSA for $30 \mathrm{~min}$ in room temperature, the cells in each group were supplemented with rabbit polyclonal antibody to PARP-1 and goat polyclonal antibody to ART1 at the dilution of $1: 100$ at $4{ }^{\circ} \mathrm{C}$ for overnight. The cells were then incubated with goat anti-rabbit $\mathrm{IgG}$ FITC (ZSGB-Bio) and rabbit anti-goat IgG RBITC (Wuhan Boster Biological Technology, Ltd.) for $1 \mathrm{~h}$. The distribution levels of PARP-1 and ART1 were analyzed by double-label immunofluorescence staining under a laser scanning confocal microscope (Leica TCS SP2, Leica Microsystems GmbH, Germany). The mean density of the immunofluorescence images was analyzed using Image-Pro Plus 6.0 software (IPP; Media Cybernetics, Bethesda, MD, USA).

CT26 cell transfection. The CT26 cells were transfected with a lentivirus based short-hairpin RNA (shRNA) vector with the following ART1-shRNA interference sequence: GCCAACAAAGTATACGCGGAT. CT26 cells transfected with non-target shRNA (control-shRNA) (interference sequence: TTCTCCGAACGTGTCACGT) were used as the control group. Transfection was carried according to the manufacturer's instructions (GeneChem, Shanghai, China). When the cells were in the logarithmic growth phase, they were cultured in 12-well plates at the concretion of $3 \times 10^{4}$ cells/ well. When the cells covered $50 \%$ of each well, $10 \mu 1$ lentivirus particles were added to each well. Transfection efficiency was optimized using green fluorescent protein and detected under a fluorescence microscope after 3 days. The CT26 cells transfected with non-targeted shRNA and the CT26 untransfected cells served as the controls. The effectiveness of the knockdown of ART1 was determined by reverse transcriptase (RT)-PCR and western blot analysis. 
$R T-P C R$. RNA was separately extracted from the ART1shRNA- and control-shRNA-transfected cells, as well as from the untransfected CT26 cells according to the manual provided with TRIzol reagent (Takara, Dalian, China). ART1 (target gene) and $\beta$-actin (internal control gene) expression was detected using oligonucleotide primers which were designed and produced by Sangon Biotech Company (Shanghai, China). The primers used were as follows: ART1, 5'-ACCTTCTTCGG TATCTGGACCT-3' (F1) and 5'-TAAGTTGCTGGAGA CCTGGATT-3' (R1); $\beta$-actin, 5'-ATATCGCTGCGCTGG TCGTC-3' (F1) and 5'-AGGATGGCGTGAGGGAG AGC-3' (R1). Using a one step RT-PCR kit (Takara), reverse transcription was carried out at $50^{\circ} \mathrm{C}$ for $30 \mathrm{~min}, 94^{\circ} \mathrm{C}$ for $2 \mathrm{~min}$, and extension was carried out for 30 cycles at $94^{\circ} \mathrm{C}$ for $30 \mathrm{sec}, 60^{\circ} \mathrm{C}$ for $30 \mathrm{sec}$ and $72^{\circ} \mathrm{C}$ for $25 \mathrm{sec}$. Finally, the amplification products were electrophoresed on $2 \%$ agarose gels (Genview, Tallahasses, FL, USA).

Western blot analysis. Adherent cells were collected by scraping into EP tubes after being washed with PBS. The cells were lysed with lysis buffer (Beyotime, Shanghai, China) for $30 \mathrm{~min}$ on ice, followed by centrifugation at 12,000 rpm for 5 min in centrifuge tubes (Thermo Fisher Scientific, Waltham, UK). Protein concentration was detected using a BCA protein assay kit (Beyotime). The proteins were then loaded in 6 or $10 \%$ polyacrylamide gels; separated by electrophoresis in the range of proper voltage; and transferred onto polyvinylidene fluoride membranes (Millipore, Billerica, MA, USA). Skimmed milk (5\%) was used to block heterogenetic antigen on the membranes for $2 \mathrm{~h}$. All blots were respectively incubated in $4^{\circ} \mathrm{C}$ overnight with primary antibodies against PARP-1, ART1, NF- $\kappa$ B (Bioworld Technology, Co., Ltd., St. Louis Park, MN, USA) and $\beta$-actin (Wuhan Boster Biological Technology, Ltd.) at individual dilutions of 1:1,000, 1:500, 1:500 and 1:500. Secondary antibodies, peroxidaseconjugated anti-goat or rabbit $\operatorname{IgG}$, were then added, followed by incubation for $1 \mathrm{~h}$ at room temperature. After being washed 3 times, the blots were dipped into BeyoECL Plus (Beyotime) for exposure and imaged (Bio-Rad, Hercules, CA, USA). Finally, Quantity One software (Bio-Rad) was used to for the densitometric analysis of proteins on each blot.

Statistical analysis. The results from RT-PCR and western blot analysis are presented as the means \pm standard deviation (SD). Using SPSS 18.0 software (SPSS, Chicago, IL, USA), differences between different groups were analyzed using one-way ANOVA and the Wilcoxon test and correlation analysis was carried out using Spearman's correlation analysis. A P-value $<0.05$ was considered to indicate a statistically significant difference.

\section{Results}

Expression of PARP-1 and ART1 in colorectal carcinoma. PARP-1 positive staining was observed in the tumor cell nuclei, closer to the nucleus, and ART1 staining was observed in the cytomembrane and cytoplasm of the carcinoma cells (Fig. 1). In the 63 cases of colorectal carcinoma, the positive ratio of PARP-1 and ART1 was $95.24 \%(60 / 63)$ and $63.49 \%$ (40/63), respectively, which was significantly higher than the
Table I. Expression of PARP-1 and ART1 in colorectal carcinoma and control colonic mucosa.

\begin{tabular}{|c|c|c|c|c|c|c|}
\hline & \multicolumn{2}{|c|}{ ART1 } & \multirow[b]{2}{*}{ P-value } & \multicolumn{2}{|c|}{ PARP-1 } & \multirow[b]{2}{*}{ P-value } \\
\hline & - & + & & - & + & \\
\hline Carcinoma & 7 & 56 & $<0.001$ & 3 & 60 & $<0.001$ \\
\hline Control group & 8 & 2 & & 9 & 1 & \\
\hline
\end{tabular}

ART1, ADP-ribosyltransferase 1; PARP-1, poly(ADP-ribose) polymerase-1.

Table II. Immunohistochemical expression of PARP-1 and ART1 in colorectal cancer.

\begin{tabular}{lrrr}
\hline & \multicolumn{2}{c}{ PARP-1 } & \\
\cline { 2 - 3 } ART1 & - & + & $\mathrm{r}$ \\
\hline- & 2 & 1 & $0.65^{\mathrm{a}}$ \\
+ & 1 & 60 & \\
\hline
\end{tabular}

( ${ }^{\mathrm{P}<0.01)}$. PARP-1, poly(ADP-ribose) polymerase-1; ART1, ADPribosyltransferase $1 ; \mathrm{r}$, correlation.

positive ratio of PARP-1 (4.76\%) and ART1 (36.51\%) in the control colonic mucosa samples $(\mathrm{P}<0.05)$ (Table I). A positive correlation was observed in the expression of PARP-1 and ART1 in the colorectal carcinoma tissues $(r=0.65)$ (Table II) $(\mathrm{P}<0.05)$.

Effects of MIBG or 5-AIQ on the expression of ART1 and PARP-1 in CT26 cells. PARP-1 and ART1 double-label immunofluoresence staining revealed that PARP-1 and ART1 were located in the cytoplasm and nucleus separately (Fig. 2A). A significant decrease in PARP and ART1 expression was observed in the group treated with MIBG compared to the untreated group $(\mathrm{P}<0.05)$. However, in the group treated with 5-AIQ, the expression of PARP was lower than that in untreated group $(\mathrm{P}<0.05)$; however, the expression of ART1 did not differ between the 2 groups $(\mathrm{P}>0.05)$ (Fig. 2B).

Knockdown of ART1 expression by transfection with shRNA lentivirus in CT26 cells. Immunofluorescence images of the transfected cells revealed that the transfection efficiency reached $80 \%$ following transfection with lentivirus particles (Fig. 3A). Compared to the control-shRNA-transfected and untransfected cells, a significant decrease in ART1 mRNA and protein expression was observed in the ART1-shRNAtransfected cells by RT-PCR and western blot analysis (Fig. 3B-E). However, the difference in ART1 mRNA and protein expression between the control-shRNA-transfected and untransfected cells was not significant ( $\mathrm{p}>0.05)$.

Effect of silencing ART1 on the expression of PARP-1 and $N F-\kappa B$ in CT26 cells. The results from western blot analsyis 


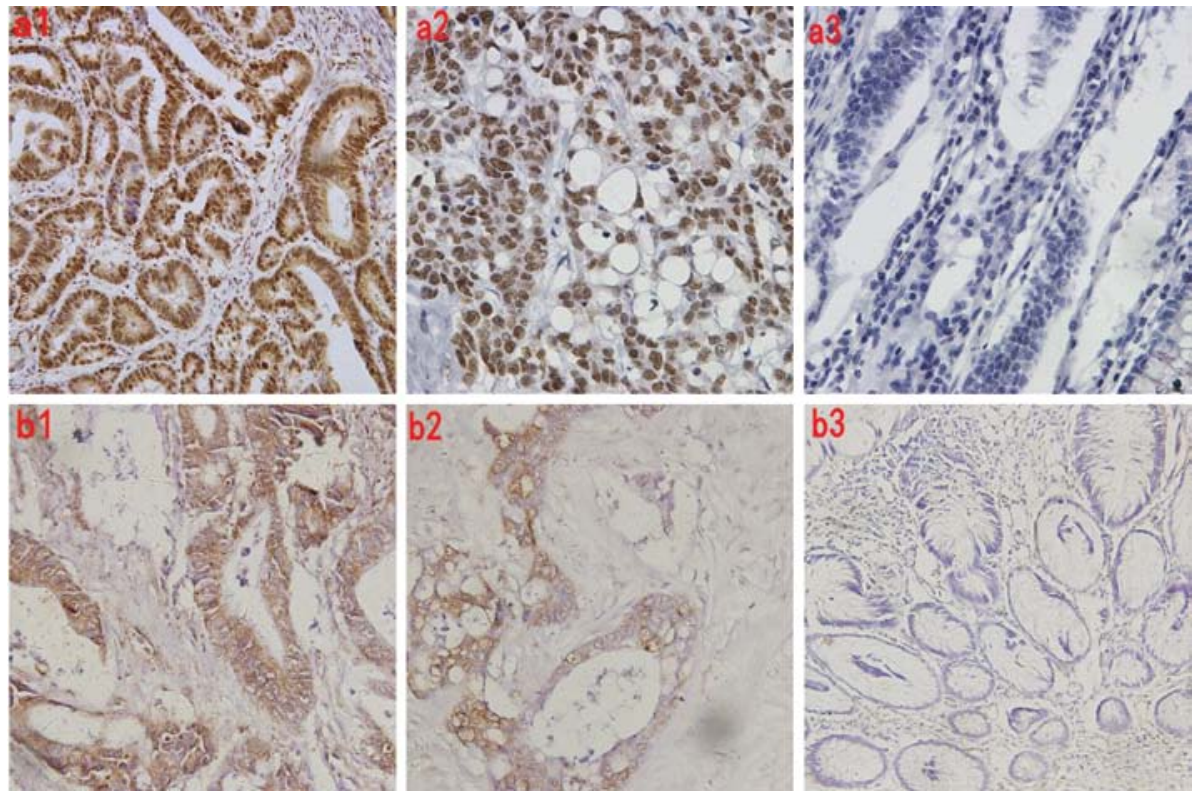

Figure 1. Expression of poly(ADP-ribose) polymerase-1 (PARP-1) and ADP-ribosyltransferase 1 (ART1) in colon carcinoma tissues. The positive expression of PARP-1 in colon carcinoma (a1) (x200), (a2) (x400), and the expression of ART1 in colon carcinoma (b1) (x200), (b2) (x400). The control group showed (a3) PARP-1 and (b3) ART1 negative expression.

A
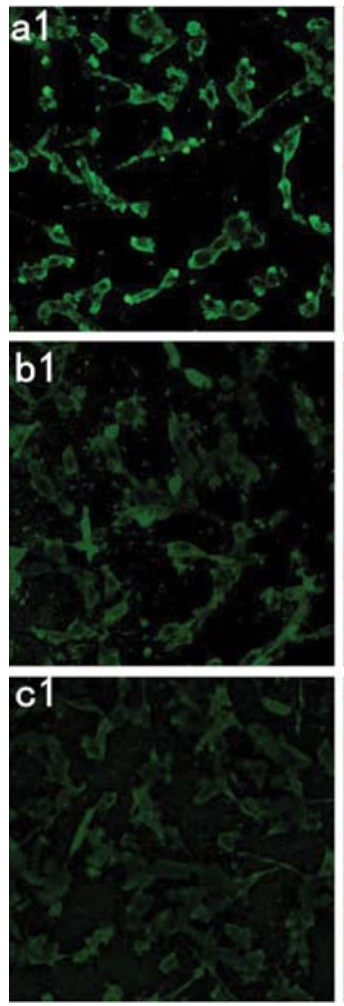
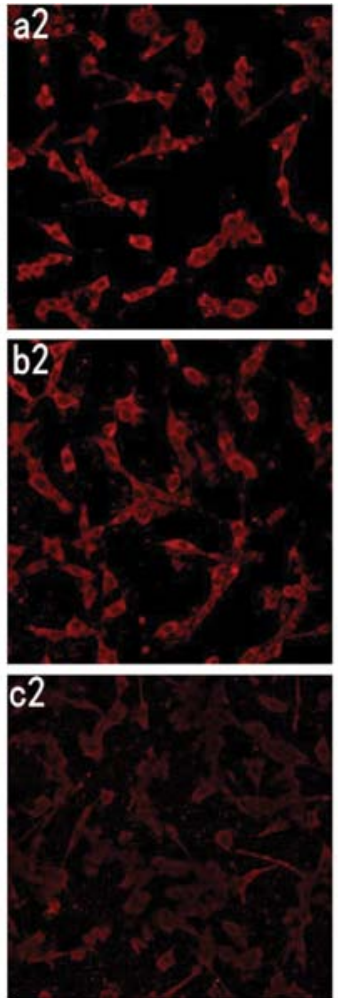
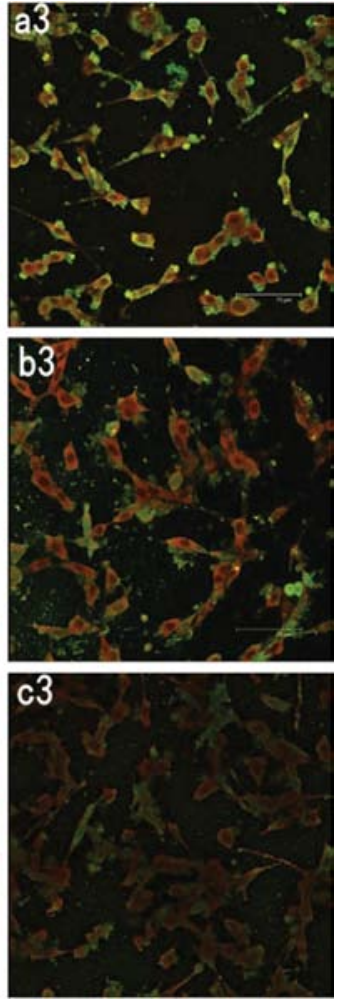

B

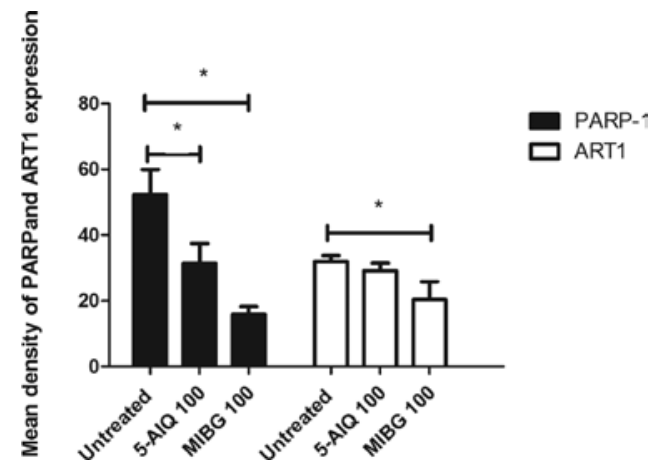

Figure 2. (A) Immunofluorescence expression of poly(ADP-ribose) polymerase-1 (PARP-1) and ADP-ribosyltransferase 1 (ART1) in the different treatment groups of CT26 cells. (a1-a3) untreated group; (b1-b3) group treated with $100 \mu \mathrm{M}$ 5-aminoisoquinolinone (5-AIQ); (c1-c3) group treated with $100 \mu \mathrm{M}$ meta-iodobenzylguanidine (MIBG). (a1, b1 and c1) FITC staining shows PARP-1 expression; (a2, b2 and c2) RBICT staining shows ART1 expression. (a3, b3 and c3) FITC and RBITC staining showing both PARP-1 and ART1 expression. (B) Mean density of PARP-1 and ART1 expression detected by IPP in the untreated group of CT26 cells, the group of cells treated with $100 \mu \mathrm{M}$ 5-AIQ and the group of cells treated with $100 \mu \mathrm{M}$ MIBG. 
A
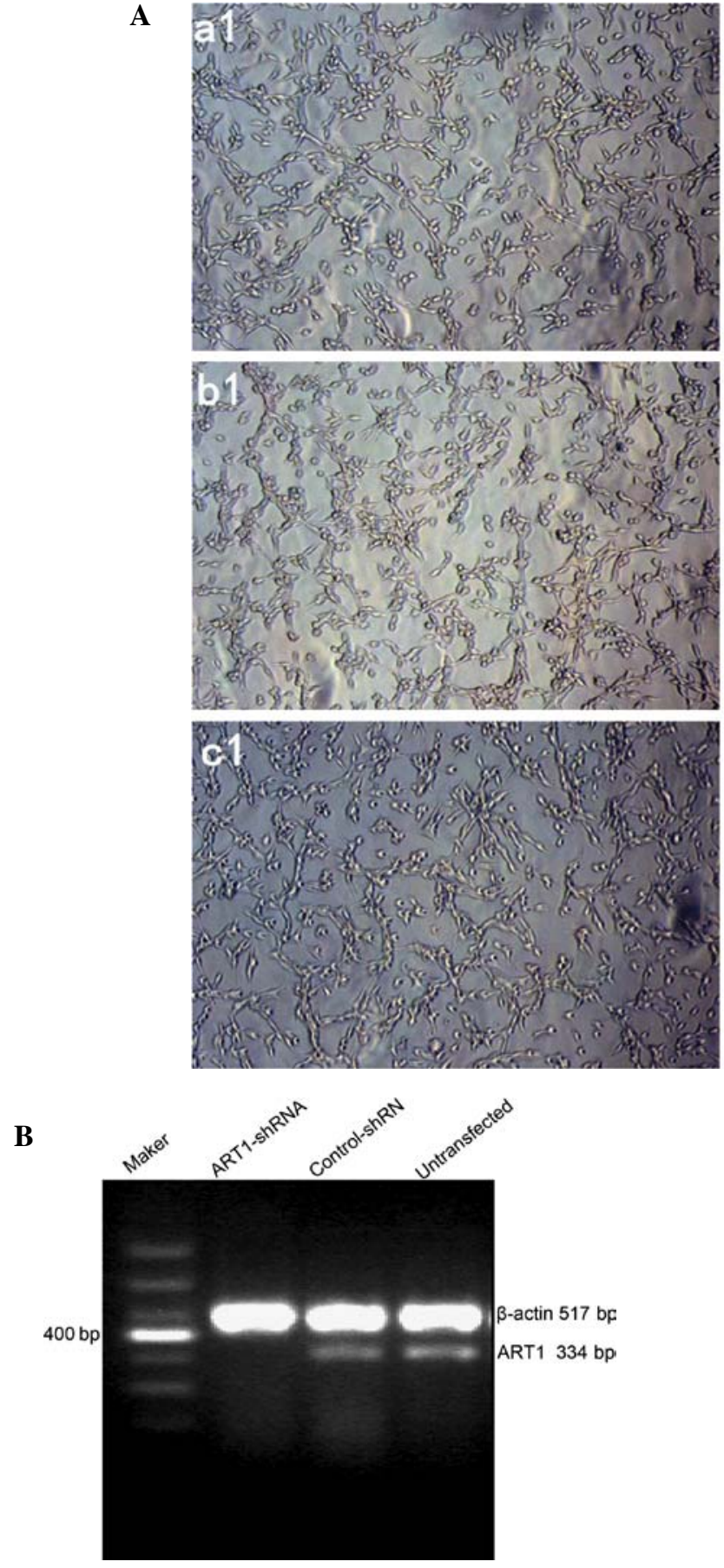

D
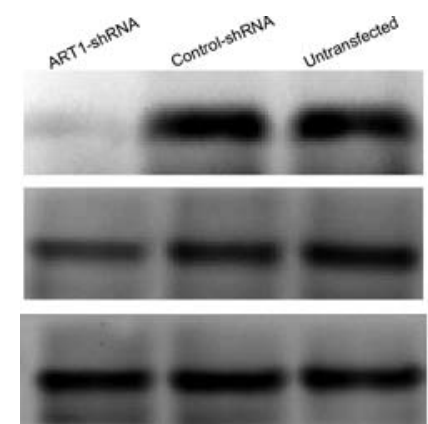

ART1 $(40 \mathrm{kDa})$

PARP $(116 \mathrm{kDa})$

$\beta$-actin $(43 \mathrm{kDa})$
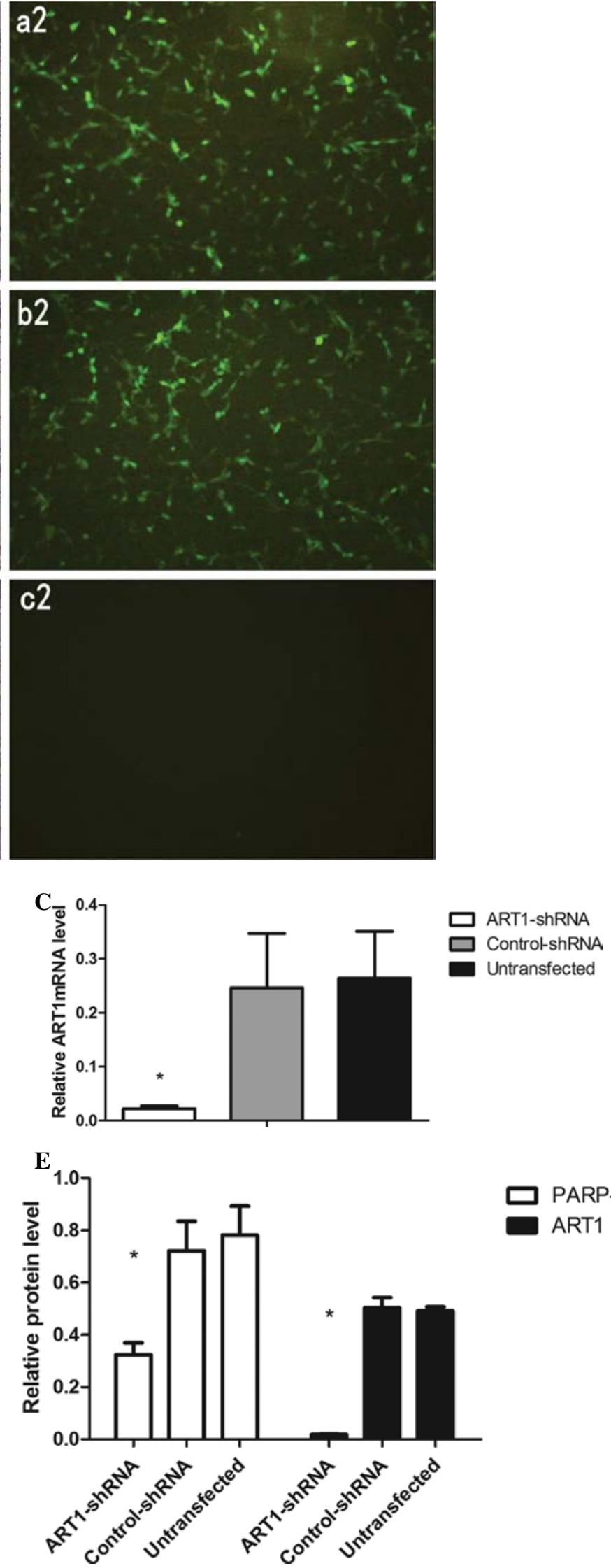

Figure 3. (A) CT26 cells transfected with $10 \mu$ of ADP-ribosyltransferase 1 (ART1)-shRNA lentivirus particles (a2) and control-shRNA lentivirus particles (b2) containing TurboGFP (x400), compared with white images of ART1-shRNA-transfected cells (a1) and control-shRNA-transfected cells (b1) showing that the number of transfected cells reach approximately $80 \%$. The untransfected cells (c1) show no fluorescence under a fluorescence microscope (c2). (B and C) Effect of the knockdown ART1 by shRNA in CT26 cells. Compared with the control-shRNA-transfected and the untransfected cells, the ART1-shRNA-transfected cells showed a decrease in ART1 expression by RT-PCR ("p<0.05). (D and E) The inhibition of ART1 altered the expression of ART1 and poly(ADP-ribose) polymerase-1 (PARP-1) in the different groups of CT26 cells. Using western blot analysis, we detected a decrease in the expression of ART1 and PARP-1 in the ART1-shRNA-transfected cells compared with the control-shRNA-transfected and untransfected CT26 cells ( $\left.{ }^{*} \mathrm{p}<0.05\right)$. $\beta$-actin was used as an internal control. 
A
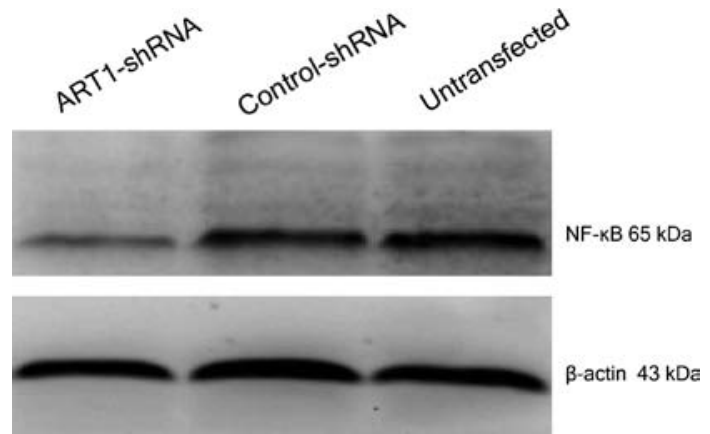

B

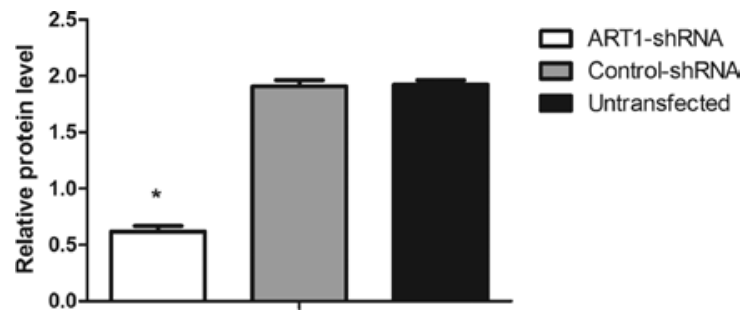

Figure 4. (A and B) Inhibition of ADP-ribosyltransferase 1 (ART1) altered the expression of NF- $\kappa \mathrm{B}$ in the different groups of CT26 cells. Using western blot analysis, we detected a decrease in the expression of NF- $\kappa \mathrm{B}$ in the ART1-shRNA-transfected cells compared to the control-shRNA-transfected and untransfected CT26 cells $\left({ }^{*} \mathrm{p}<0.05\right)$.

revealed that the expression of PARP-1 and NF- $\mathrm{kB}$ was lower in the ART1-shRNA-transfected cells compared to the untransfected cells or the control-shRNA-transfected cells $(\mathrm{p}<0.05)$ (Figs. 3D and E, 4A and B). However, the difference in PARP-1 and NF- $\mathrm{KB}$ expression between the control-shRNA-transfected and untransfected cells was not significant $(\mathrm{p}>0.05)$.

\section{Discussion}

In a recent study, Kato et al reported that the absence of ADP-ribosylarginine hydrolase (ARH1), arginine-specific mono-ADP-ribosylation hydrolase, may increase the frequency and extent of tumorigenesis in mice, including lung adnocarcinoma, hepatocellular carcinoma and lymphoma (31). They suggested that the levels of protein ADP-ribosylation controlled by ARH1 may suppress carcinoma. ARH1 and ART1 collectively participate in the reversible modification of proteins in the cycle of mono-ADP-ribosylation, to control the levels of arginine ADP-ribosylation on substrate proteins. ARH1, which has an opposite function to ART1, cleaves $\alpha$-ADP-ribose-arginine to regenerate the arginine-guanidino group $(1,32)$. The knockdown of ARH1 in cells has been shown to increase the ADP-ribose-arginine content (31). Hence, the suppression of carcinogenesis by ARH1 suggests that ART1 has a positive effect on carcinogenesis. In the present study, our results revealed a higher expression of ART1 in human colon carcinoma tissues compared to normal colonic mucosa. This suggests that ART1 plays a role in colon carcinoma.

Thyberg et al previously demonstrated that inhibitors of poly-ADP-ribosylation (hexamethylenebisacetamide, HMBA) and mono-ADP-ribosylation (MIBG) suppress the phenotypic modulation and proliferation of SMCs. Both inhibitors of ADP-ribosylation inhibited SMC proliferation and suppressed DNA synthesis. However, compared to the inhibition of mono-ADP-ribosylation, the inhibition of poly-ADP-ribo- sylation significantly reduced the expression of transcripts for differentiation markers and matrix metalloproteinases, which are involved in SMC migration and proliferation (17). However, to our knowledge, to date, there are no studies available on the correlation between poly-ADP-ribosylation and mono-ADP-ribosylation in colon carcinoma. Thus, in this study, we examined the activity of PARP-1 and ART1 to elucidate the correlation between poly-ADP-ribosylation and mono-ADP-ribosylation in colon carcinoma. The results revealed a positive correlation between the expression of ART1 and PARP-1 in colon carcinoma. To further confirm the correlation between these 2 enzymes, we used an inhibitor of PARP-1 and ART1 and observed the changes in the expression of PARP-1 and ART1. MIBG (an inhibitor of ART1) simultaneously inhibited the expression of PARP-1 and ART1, but 5-AIQ (an inhibitor of PARP-1) only inhibited the expression of PARP-1. Furthermore, after silencing the $A R T 1$ gene, the levels of PARP-1 and ART1 in the CT26 cells decreased. However, the mechanisms involved require further clarification.

Yau et al indicated that MIBG inhibits the phosphorylation of the Rho effector, PRK1/2, a downstream effector of Rho, suggesting that mono-ADP-ribosylation participates in a Rho-dependent signaling pathway (33). Rho proteins are members of the Ras superfamily of GTPases, which includes 9 members, RhoA, RhoB, RhoC, RhoG, RhoE, CDC42, Rac-1, Rac-2 and TC10 (34). Our data demonstrated that the expression of RhoA was reduced in the ART1-shRNA-transfected CT26 cells compared with the untransfected CT26 cells, (data not shown). Accordingly, RhoA lies downstream of mono-ADPribosylation mediated by ART1. Studies have demonstrated that the inhibition of RhoA has a negative effect on NF- $\mathrm{KB}$ activity by decreasing phospho-I $\kappa \mathrm{B} \alpha$ levels in prostate carcinoma cells (35); and may also suppress TNF $\alpha$-induced NF- $\mathrm{\kappa B}$ activation, decreasing the invasive and metastatic ability of breast carcinoma cells (36). Su et al revealed a positive correlation between NF- $\mathrm{KB}$ and RhoA in gastric carcinoma (37). It is also known that RhoA is involved in the regulation of

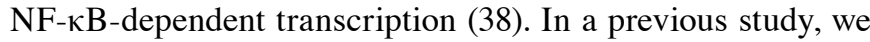
demonstrated that the inhibition of NF- $\mathrm{KB}$, using the inhibitor, pyrrolidinedithiocarbamic acid (PDTC) decreased the expressoin of PARP-1 in human colon carcinoma Lovo cells compared to untreated Lovo cells. Therefore, the inhibtion of NF- $\kappa$ B may inhibit the expression of PARP-1 through a feedback mechanism (26). We hypothesized that the ART1-mediated activity of RhoA may affect NF- $\kappa B$ transcription, regulating the expression of PARP-1 through the feedback mechanism of NF- $\mathrm{KB}$ to PARP-1. In this study, confirming our hypothesis, we detected the reduced expression of NF- $\mathrm{KB}$ following the transfection of CT26 cells with ART1-shRNA. Therefore, it can be concluded that ART1 mediates the expression of PARP-1 by altering the expression of NF- $\mathrm{KB}$. However, the mechanisms behind the effects of the ART1-mediated activity of RhoA require further clarification.

In brief, our data demonstrate that PARP-1 expression is affected by ART1 in colon carcinoma. The proposed mechanism behind this effect may involve arginine monoADP-ribosylation mediated by ART1 which negatively affects the expression of RhoA and NF- $\kappa \mathrm{B}$ activity, and sequentially decreases PARP-1 expression. However, further studies are required to elucidate the precise mechanisms involved. Further 
studies investigating the mechanisms behind the correlation between PARP-1 and ART1 may help to clarify the role of ADP-ribosylation in the development of colon carcinoma.

\section{Acknowledgements}

This study was supported by the Ministry of Education Specialized Research Fund for the Doctoral Program of Higher Education (Grant no. 20105503110009) and the Science and Technology Project of the Education Commission of Chongqing (Grant no. KJ110322).

\section{References}

1. Hassa PO, Haenni SS, Elser M and Hottiger MO: Nuclear ADP-ribosylation reactions in mammalian cells: where are we today and where are we going? Microbiol Mol Biol Rev 70: 789-829, 2006.

2. Moss J, Balducci E, Cavanaugh E, et al: Characterization of NAD: arginine ADP-ribosyltransferases. Mol Cell Biochem 193: 109-113, 1999

3. Zolkiewska A, Nightingale MS and Moss J: Molecular characterization of NAD: arginine ADP-ribosyltransferase from rabbit skeletal muscle. Proc Natl Acad Sci USA 89: 11352-11356, 1992.

4. Corda D and Di Girolamo M: Functional aspects of protein mono-ADP-ribosylation. EMBO J 22: 1953-1958, 2003.

5. Corda D and Di Girolamo M: Mono-ADP-ribosylation: a tool for modulating immune response and cell signaling. Sci STKE 2002 pe53, 2002

6. Helleday T, Bryant HE and Schultz N: Poly(ADP-ribose) polymerase (PARP-1) in homologous recombination and as a target for cancer therapy. Cell Cycle 4: 1176-1178, 2005.

7. Hao LX, Wang YL and LI YY: Correlation of PARP expression with P-selectin and ICAM-1 expression in colorectal carcinoma. Basic Clin Med 26: 882-887, 2006.

8. Li M, Cai L and Wang Y: Effect of poly(ADP-ribose) polymerase inhibition on liver metastasis of mouse colorectal carcinoma CR26 cell line in vivo. Acta Acad Med Mil Tert 30: 237-240, 2008.

9. Wang YL and Hao LX: Effect of 5-aminoisoquinolinone on the adhesion of colon carcinoma. Chin J Cancer Res 19: 119-123, 2007.

10. Qin Y Wang YL and Li YY: Effect of poly(ADP-ribose) polymerase inhibition on liver metastasis of mouse colorectal carcinoma CT26 cell line in vivo. Acta Acad Med Mil Tert 29: $1621-1624,2008$

11. Wang J, Nemoto E, Kots AY, Kaslow HR and Dennert G: Regulation of cytotoxic $\mathrm{T}$ cells by ecto-nicotinamide adenine dinucleotide (NAD) correlates with cell surface GPI-anchored/arginine ADP-ribosyltransferase. J Immunol 153 4048-4058, 1994

12. Wang J, Nemoto E and Dennert G: Regulation of CTL by ecto-nictinamide adenine dinucleotide (NAD) involves ADP-ribosylation of a p56lck-associated protein. J Immunol 156 : 2819-2827, 1996.

13. Okamoto S, Azhipa O, Yu Y, Russo E and Dennert G: Expression of ADP-ribosyltransferase on normal $\mathrm{T}$ lymphocytes and effects of nicotinamide adenine dinucleotide on their function. J Immunol 160: 4190-4198, 1998.

14. Adriouch S, Ohlrogge W, Haag F, Koch-Nolte F and Seman M: Rapid induction of naive $\mathrm{T}$ cell apoptosis by ecto-nicotinamide adenine dinucleotide: requirement for mono(ADP-ribosyl) transferase 2 and a downstream effector. J Immunol 167: 196-203, 2001.

15. Akai T, Nabeya Y, Yahiro K, et al: Helicobacter pylori induces mono-(adenosine 5'-diphosphate)-ribosylation in human gastric adenocarcinoma. Int J Oncol 29: 965-972, 2006.

16. Yang L, Wang Y, Sheng Y, Xiong W, Xu J, Tang Y and Li X: The correlation of ART1 expression with angiogenesis in colorectal carcinoma and it relationship with VEGF and integrin $\alpha \mathrm{V} \beta 3$ expressions. Basic Clin Med 9: 1064-1069, 2012.
17. Thyberg J, Hultgårdh-Nilsson $\mathrm{A}$ and Kallin B: Inhibitors of ADP-ribosylation suppress phenotypic modulation and proliferation of smooth muscle cells cultured from rat aorta. Differentiation 59: 243-252, 1995.

18. Brac T and Ebisuzaki K: PolyADP ribosylation and Friend erythroleukemic-cell differentiation: action of poly(ADP-ribose) polymerase inhibitors. Differentiation 34: 139-143, 1987.

19. Colon-Otero G, Sando JJ, Sims JL, McGrath E, Jensen DE and Quesenberry PJ: Inhibition of hemopoietic growth factor-induced proliferation by adenosine diphosphate-ribosylation inhibitors. Blood 70: 686-693, 1987.

20. Francis G, Gray D, Berney J, Wing M, Guimaraes $\mathbf{J}$ and Hoffbrand A: Role of ADP-ribosyltransferase in differentiation of human granulocyte-macrophage progenitors to the macrophage lineage. Blood 62: 1055-1062, 1983.

21. Kuo ML, Chau YP, Wang JH and Shiah SG: Inhibitors of poly(ADP-ribose) polymerase block nitric oxide-induced apoptosis but not differentiation in human leukemia HL-60 cells. Biochem Biophys Res Commun 219: 502-508, 1996.

22. Terada M, Fujiki H, Marks PA and Sugimura T: Induction of erythroid differentiation of murine erythroleukemia cells by nicotinamide and related compounds. Proc Natl Acad Sci USA 76: 6411-6414, 1979.

23. Luo J, Kamata $\mathrm{H}$ and Karin M: IKK/NF-kappaB signaling: balancing life and death - a new approach to cancer therapy. J Clin Invest 115: 2625-2632, 2005.

24. Karin M and Ben-Neriah Y: Phosphorylation meets ubiquitination: the control of $\mathrm{NF}-\kappa \mathrm{B}$ activity. Annu Rev Immunol 18: $621-663,2000$

25. Virág L and Szabó C: The therapeutic potential of poly(ADP-ribose) polymerase inhibitors. Pharmacol Rev 54: 375-429, 2002.

26. Pan J, Fauzee NJ, Wang YL, et al: Effect of silencing PARG in human colon carcinoma LoVo cells on the ability of HUVEC migration and proliferation. Cancer Gene Ther 19: 715-722, 2012.

27. LoesbergC,vanRooijH, andSmetsLA:Meta-iodobenzylguanidine (MIBG), a novel high-affinity substrate for cholera toxin that interferes with cellular mono(ADP-ribosylation). Biochim Biophys Acta 1037: 92-99, 1990.

28. Mota-Filipe H, Sepodes B, McDonald M, Cuzzocrea S, Pinto R and Thiemermann C: The novel PARP inhibitor 5-aminoisoquinolinone reduces the liver injury caused by ischemia and reperfusion in the rat. Med Sci Monit 8: BR444-BR453, 2002.

29. Fromowitz FB, Viola MV, Chao S, et al: Ras p21 expression in the progression of breast cancer. Hum Pathol 18: 1268-1275, 1987.

30. Qin JM, Fu XY, Li SJ, et al: Gene and protein expressions of p28GANK in rat with liver regeneration. World J Gastroenterol 9: 2523-2527, 2003

31. Kato J, Zhu J, Liu C, et al: ADP-ribosylarginine hydrolase regulates cell proliferation and tumorigenesis. Cancer Res 71: 5327-5335, 2011.

32. Moss J, Stanley S, Nightingale M, et al: Molecular and immunological characterization of ADP-ribosylarginine hydrolases. J Biol Chem 267: 10481-10488, 1992.

33. Yau L, Litchie B, Thomas S, Storie B, Yurkova N and Zahradka P: Endogenous mono-ADP-ribosylation mediates smooth muscle cell proliferation and migration via protein kinase $\mathrm{N}$-dependent induction of c-fos expression. Eur J Biochem 270: 101-110, 2003.

34. Bourne HR, Sanders DA and McCormick F: The GTPase superfamily: conserved structure and molecular mechanism. Nature 349: 117-127, 1991

35. Hwang YS, Hodge JC, Sivapurapu N and Lindholm PF: Lysophosphatidic acid stimulates $\mathrm{PC}-3$ prostate cancer cell matrigel invasion through activation of RhoA and NF- $\mathrm{BB}$ activity. Mol Carcinog 45: 518-529, 2006.

36. Cho SG,Li D, Stafford LJ, et al: KiSS1 suppresses TNF $\alpha$-induced breast cancer cell invasion via an inhibition of RhoA-mediated NF-kB activation. J Cell Biochem 107: 1139-1149, 2009.

37. Su X, Tang Z, Li Q, et al: Expression and significance of RhoA and NF- $\kappa \mathrm{B}$ in human gastric carcinoma. Zhonghua Zhong Liu Za Zhi 33: 276-279, 2011 (In Chinese).

38. Perona R, Montaner S, Saniger L, Sanchez-Perez I, Bravo R and Lacal JC: Activation of the nuclear factor-kappaB by Rho, CDC42, and Rac-1 proteins. Genes Dev 11: 463-475, 1997. 